\title{
SHIP1 is targeted by miR-155 in acute myeloid leukemia
}

\author{
HUA XUE $^{1}$, LUO-MING HUA $^{2}$, MING GUO $^{2}$ and JIAN-MIN LUO ${ }^{1}$ \\ ${ }^{1}$ Department of Hematology, The Second Hospital of Hebei Medical University, Shijiazhuang, Hebei 050000; \\ ${ }^{2}$ Department of Hematology, Affiliated Hospital of Hebei University, Baoding, Hebei 071000, P.R. China
}

Received April 30, 2014; Accepted July 29, 2014

DOI: $10.3892 /$ or.2014.3435

\begin{abstract}
The SH2 domain-containing inositol 5'-phosphatase 1 (SHIP1) has been implicated as a suppressor of hematopoietic transformation as its activity can inhibit the PI3K/Akt signaling pathway. Reduced activity of SHIP1 has been observed in acute myeloid leukemia (AML). SHIP1 is a target of microRNA-155 (miR-155). Therefore, the aim of the present study was to investigate the role of miR-155/SHIP1 in the pathogenesis of AML. We examined the levels of SHIP1 protein and miR-155 in tissue samples of patients with AML and in AML cell lines. In addition, we investigated cell proliferation, apoptosis and expression of SHIP1/PI3K/AKT pathway molecules in the THP-1 and U937 cell lines after miR-155 inhibitor or mimics were transfected. We showed that the levels of SHIP1 protein were significantly decreased in tissue samples of patients with some subtypes of AML (M4 or M5) and in AML cell lines with concomitant overexpression of miR-155. In addition, we demonstrated that decreased expression of SHIP1 in the AML cell lines was a consequence of increased levels of miR-155 and can therefore be reversed in vitro through inhibition of miR-155, with subsequent inhibition of cell proliferation and promotion of cell apoptosis. In conclusion, expression of the SHIP1 protein is targeted by miR-155 in AML. miR-155 acts as an onco-miR, and the miR-155/SHIP1/PI3K/AKT signaling pathway could play an important role in the pathogenesis of AML.
\end{abstract}

\section{Introduction}

SH2 domain-containing inositol 5'-phosphatase 1 (SHIP1) is a member of the phosphatidylinositol phosphatase (PIPase) class that is important in the negative regulation of proliferation and survival of hematopoietic cells through its negative regulation of the PI3K/AKT signaling pathway $(1,2)$. Recent studies have shown that inactivation of SHIP1 could play a central role in certain hematologic malignancies (1-6). Inactivation of

Correspondence to: Dr Jian-Min Luo, Department of Hematology, The Second Hospital of Hebei Medical University, Shijiazhuang, Hebei 050000, P.R. China

E-mail: luojm2011@163.com

Key words: SH2 domain-containing inositol 5'-phosphatase 1, microRNA-155, acute myeloid leukemia point mutations of the SHIP1 gene and reduction in SHIP1 activity have been observed in patients with acute myeloid leukemia (AML), implicating a possible tumor-suppressor function of SHIP1 in the pathogenesis of AML (6). However, it appears that SHIP1 gene mutations are an uncommon cause of reduction in SHIP1 activity in AML (7). Several other possible explanations that could account for the loss of SHIP1 function include epigenetic modification, decreased transcription, translational repression, and increased protein breakdown. miR-155 has been shown to bind to the 3'UTR of SHIP1 mRNA and inhibit its translation (8-11). It was demonstrated that SHIP1 is a primary target of miR-155 in B cells, with high levels of miR-155 and reduced expression of SHIP1 linked to the development of acute lymphoblastic leukemia in mice $(3,12)$. Levels of miR-155 were also found to be significantly increased in human patients with diffuse large B cell lymphoma or NK/T cell leukemia with decreased SHIP1 expression $(13,14)$. Further investigation is needed to evaluate the role of miR155 and SHIP1 in the pathogenesis of AML.

In the present study, we examined the levels of SHIP1 protein and miR-155 in samples of patients with AML and in AML cell lines. In addition, we investigated cell proliferation, apoptosis and expression of SHIP1/PI3K/AKT pathway molecules in the THP-1 and U937 cell lines after miR-155 inhibitor or mimics were transfected.

\section{Materials and methods}

Bone marrow or blood samples. Bone marrow or blood samples were collected from 30 patients with AML after obtaining informed consent according to our hospital guidelines. The pathological diagnosis of AML was established according to the French-American-British (FAB) classification criteria as M1 (n=4), M2 ( $=8), M 3(n=2), M 4(n=6), M 5(n=9)$, or M6 $(n=1)$. Bone marrow mononuclear cells (BMMNCs) or peripheral blood mononuclear cells (PBMNCs) of the patients were separated by density gradient centrifugation and cryopreserved until further analysis could be performed. BMMNCs from 10 unrelated healthy volunteers were also examined for miR-155 and SHIP1 by quantitative reverse-transcription polymerase chain reaction (qRT-PCR).

Cell lines. The leukemia cell lines K562, THP-1 and U937 were purchased from the Blood Institute of Tianjin, China. Cells were cultured using RPMI-1640 medium containing $10 \%$ fetal 
bovine serum (FBS), $2 \mathrm{mM}$ glutamine, and streptomycin in a humidified incubator at $37^{\circ} \mathrm{C}$ in $5 \% \mathrm{CO}_{2}$. Cells were used for experimentation at a density of $10^{6}-10^{7} / 1$.

Cell transfections. U937 and THP-1 cells were transfected using X-tremeGENE siRNA transfection reagent (Roche, $\mathrm{CH})$ according to the manufacturer's protocol. Briefly, cells were cultured in 6 -well plates. Transfection reagent 1 was prepared by mixing $90 \mu \mathrm{l}$ of Opti-MEM medium and $10 \mu \mathrm{l}$ of X-tremeGENE siRNA transfection reagent. Additionally, reagent 2 containing siRNA ( $2 \mu \mathrm{g}$ of inhibitor) and $100 \mu \mathrm{l}$ of Opti-MEN medium was prepared. Reagent 2 was mixed with reagent 1 and incubated for $30 \mathrm{~min}$. This mixture was then carefully added to the plated cells to obtain a final concentration of miR-155 mimics or miR-155 siRNA of $50 \mathrm{nmol} / 1$. Cells were cultured for $48 \mathrm{~h}$ and then collected for analysis of gene expression and subsequent experiments. Cells used for the blank and negative control groups were treated with only serum-free RPMI-1640 medium or with control reagent (at a final concentration of $50 \mathrm{nmol} / \mathrm{l}$ ), respectively.

Measurement of apoptosis. Cellular apoptosis was measured by flow cytometry, according to the following protocol, $48 \mathrm{~h}$ after transfection. Briefly, $\sim 5-10 \times 10^{4}$ resuspended cells were collected by centrifugation at $500 \mathrm{x}$ g for $5 \mathrm{~min}$ and resuspended again in $195 \mu \mathrm{l}$ of Annexin V-FITC. A total of $5 \mu \mathrm{l}$ of Annexin V-FITC solution was added to the cells, mixed gently, and incubated in the dark at room temperature. Cells were collected again by centrifugation and resuspended in $190 \mu \mathrm{l}$ of Annexin V-FITC. A total of $10 \mu \mathrm{l}$ of propidium iodide staining solution was added and mixed, and apoptosis measurement was performed by flow cytometry. Data were analyzed using FACS Diva software (BD Biosciences, San Jose, CA, USA).

Measurement of cell proliferation. Cells were collected $48 \mathrm{~h}$ after transfection by centrifugation at $500 \mathrm{x} \mathrm{g}$ for $5 \mathrm{~min}$, resuspended, and added to 96 -well plates $(100 \mu 1 /$ well, approximately $10^{4}$ cells/well). This time point was defined as zero (0). Then, $10 \mu \mathrm{l}$ of CCK-8 was added to the wells separately at 24,48 and $72 \mathrm{~h}$, and the absorbance was measured at $450 \mathrm{~nm}$.

RNA isolation and PCR. Total RNA was extracted from cells using TRIzol reagent (Invitrogen, Carlsbad, CA, USA) following the manufacturer's protocol. The quality of total RNA was assessed using a NanoDrop 2000 Spectrophotometer (Thermo Fisher Scientific Inc., Waltham, MA, USA).

Measurement of miR-155 gene expression. Total RNA $(2 \mu \mathrm{g})$ was reversed-transcribed using an All-in-One miRNA qRT-PCR detection system (Invitrogen) according to the manufacturer's protocol. All-in-One miRNA qRT-PCR detection system kits were used to measure gene expression using specific sets of primers (Table I) according to the protocol (Invitrogen). All reactions were performed with the ABI Prism 7000 sequence detection system instrument (Applied Biosystems). Gene expression was normalized to the U6 gene, and the data were analyzed using the comparative $2^{-\Delta \Delta \mathrm{Ct}}$ method.

Measurement of SHIP-1 gene expression. Total RNA $(2 \mu \mathrm{g})$ was analyzed for gene expression using One-Step qRT-PCR
Table I. Primers and sequences of miR-155 mimics, inhibitors and negative control.

Primers Sequence (5'-3')

miR-155-5p mimics

UUAAUGCUAAUCGUGAUAGGGGU CCCUAUCACGAUUAGCAUUAAUU

Negative control for markup mimics UUCUCCGAACGUGUCACGUTT miR-155-5p inhibitor ACGUGACACGUUCGGAGAATT

Negative control ACCCCUAUCACGAUUAGCAUUAA for miR-155-5p inhibitor CAGUACUUUUGUGUAGUACAA

Table II. Primers for real-time PCR.

\begin{tabular}{ll}
\hline Primers & \multicolumn{1}{c}{ Sequence } \\
\hline SHIP1 & F: 5'-GCGTGCTGTATCGGAATTGC-3' \\
& R: 5'-TGGTGAAGAACCTCATGGAGAC-3' \\
GAPDH & F: 5'-ACCACAGTCCATGCCATCACT-3' \\
& R: 5'-TCCACCACCCTGTTGCTGTA-3' \\
miR-155 & F: 5'-CCCCTATCACGATTAGCATTAA-3' \\
U6 & F: 5'-GCAGGGGCCATGCTAATCTTCTCTGTATCG-3'
\end{tabular}

F, forward; R, reverse.

kits according to the manufacturer's protocol. Briefly, $25 \mu 1$ of 2X Quant One-Step SYBR qRT-PCR Master Mix, $2.5 \mu 1$ of $2.5 \mathrm{U} / \mu 1$ Hot Master Taq polymerase, $0.4 \mu \mathrm{l}$ of Quant Rtase, $2 \mu \mathrm{l}$ of the forward primer $(10 \mathrm{pM}), 2 \mu 1$ of the reverse primer $(10 \mathrm{pM})$ (both by Invitrogen), and $2 \mu \mathrm{g}$ of total RNA were mixed and double-distilled water was added to reach a final volume of $50 \mu \mathrm{l}$. The primer sequences of the SHIP1 gene are listed in Table II. All reactions were performed in the ABI Prism 7000 sequence detection system. Gene expression was normalized to the GAPDH gene, and the data were analyzed using the comparative $2^{-\Delta \Delta \mathrm{Ct}}$ method.

Western blotting. Cells were digested and proteins were extracted in RIPA lysis buffer. The concentration of proteins was measured by the Bradford method according to the standard protocol. A total of $20 \mu \mathrm{g}$ of total protein extract was electrophoresed on polyacrylamide/sodium dodecyl sulfate (SDS) gels and transferred by electroblotting onto polyvinylidene fluoride (PVDF) membranes. The membranes were blocked for $1 \mathrm{~h}$ in $5 \%(\mathrm{w} / \mathrm{v})$ nonfat milk before incubation with appropriate dilutions of the primary antibodies against SHIP-1, AKT, p-AKT and actin (all at 1:1,000; from Cell Signaling Technology, Boston, MA, USA) at $4^{\circ} \mathrm{C}$ overnight. The membranes were then incubated with the corresponding secondary antibodies $(1: 10,000 ;$ Zymed). The blots were developed using the enhanced chemiluminescence (ECL) system (Immobilon Western; Millipore, Billerica, MA, USA) and were analyzed using Quantity One Software (Bio-Rad 

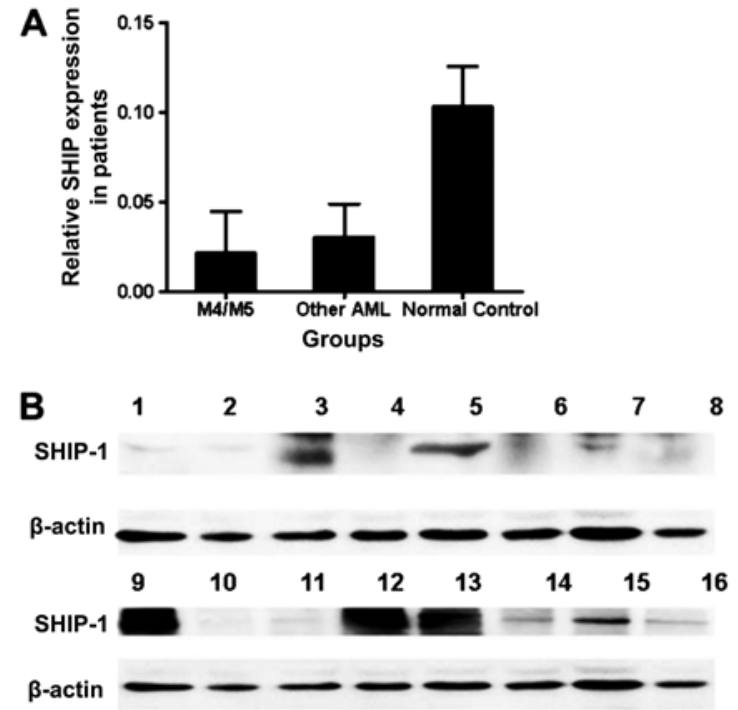
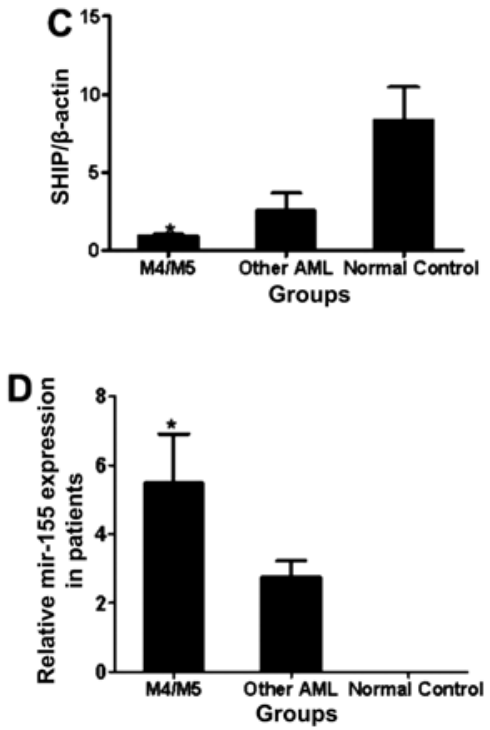

Figure 1. Expression of miR-155 and SHIP1 in bone marrow cells from the subjects. (A) Expression of SHIP1 at the mRNA level in tissue samples from patients with M4 or M5 AML ( $\mathrm{n}=15$ ), with other types of AML according to FAB classification except M4 or M5 (other AML) ( $\mathrm{n}=15)$ and in normal controls ( $\mathrm{n}=10)$. "P<0.05, M4/M5 vs. other AML. (B) SHIP1 protein levels in the tissue samples from patients and normal controls. Lanes 1, 2, 10, 11 and 16 correspond to patients with M4 or M5; lanes 3, 5, 9, 12 and 13 correspond to normal controls and lanes 4, 6, 7, 8, 14 and 15 correspond to patients with other AML. (C) SHIP1 protein levels in tissue samples from patients with M4 or M5, with other AML and in normal controls. "P<0.05, M4/M5 vs. other AML. (D) Relative gene expression of miR-155 at the mRNA level in tissue samples from patients with M4 or M5, with other AML and in normal controls. ${ }^{*} \mathrm{P}<0.05$, M4/M5 vs. other AML.
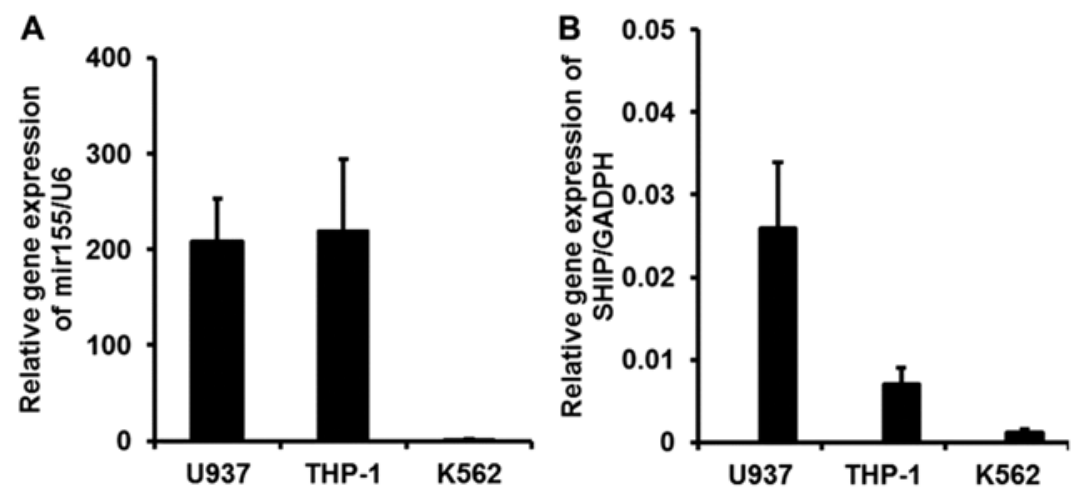

Figure 2. Expression of miR-155 and SHIP1 in AML cell lines. Relative gene expression of (A) miR-155 and (B) SHIP/GADPH in the THP1, U937 and K562 cell lines.

Laboratories Inc., Hercules, CA, USA). Protein contents were normalized to the actin level.

Data analyses. Data are expressed as the means \pm SD. Statistical analyses of experimental groups were performed by Student's t-test or one-way analysis of variance (ANOVA). The level of statistical significance was set at $\mathrm{P}<0.05$. Analyses were performed using SPSS 13.0 for Windows software (SPSS, Chicago, IL, USA).

\section{Results}

Expression of miR-155 and SHIPl in patients with AML. The SHIP1 protein content was significantly lower in the tissue samples from AML patients when compared with that in the controls $(\mathrm{P}<0.05)$. No differences were found in the expression of SHIP1 at the mRNA level among the 30 patients with different subtypes of AML according to the FAB classification (P>0.05, Fig. 1A). However, the levels of SHIP1 protein varied greatly. A much lower expression than the average level of SHIP1 protein was detected in 15 patients classified as FAB-AML-M4 or FAB-AML-M5 compared with the other subtypes of AML $(\mathrm{P}<0.05$, Fig. $1 \mathrm{~B}$ and $\mathrm{C})$. The average level of miR-155 was significantly higher in the 30 AML patients compared with the average level in the healthy controls $(\mathrm{P}<0.05)$. Additionally, the levels of miR-155 were significantly higher in patients with AML subtype M4 or M5 compared with the other AML subtypes $(\mathrm{P}<0.05$, Fig. 1D).

Expression of miR-155 and SHIP1 in AML cell lines. Real-time PCR demonstrated that both miR-155 and SHIP1 were expressed in the THP-1, K562, and U937 cell lines. Downregulation of SHIP1 expression in the K562 cells, which are known to be Philadelphia chromosome-positive, could be 

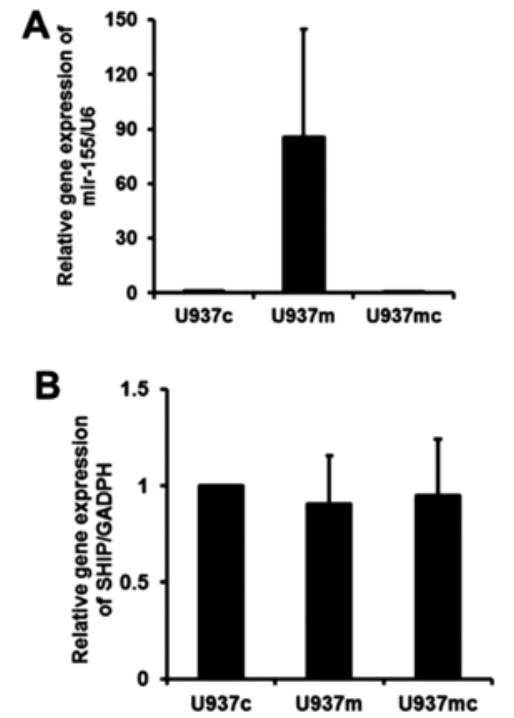
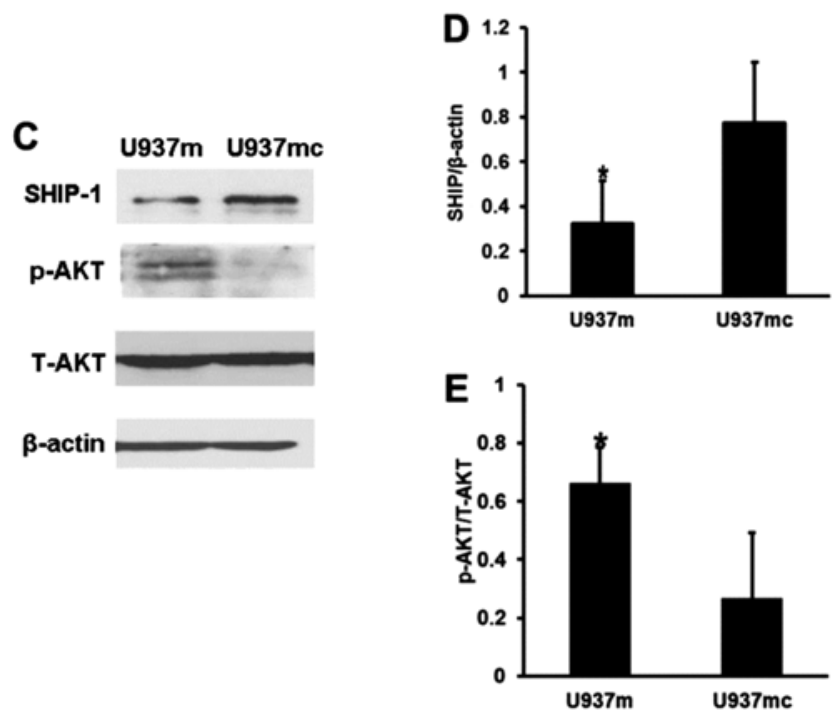

Figure 3. Expression of SHIP1, AKT, and p-AKT after miR-155 transfection. Relative gene expression of (A) miR-155 and (B) SHIP1 at the mRNA level in U937 cells without transfection (U937c), and in U937 cells transfected with the miR-155 mimics (U937m) or with the miR-155 mimic control (U937mc), (C) Protein levels of SHIP1, p-AKT and T-AKT in the U937m and U937mc cells. (D) A significantly decreased SHIP1 level and (E) increased p-AKT/T-AKT ratio were found in the U937m cells when compared with the U937mc cells. ${ }^{*} \mathrm{P}<0.05$ vs. U937mc.
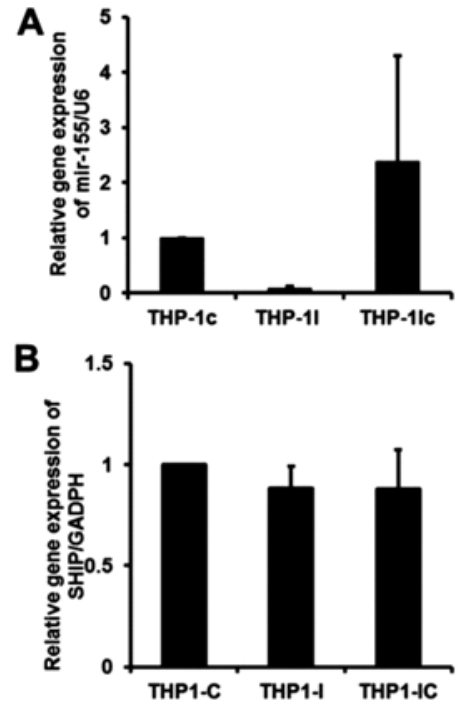
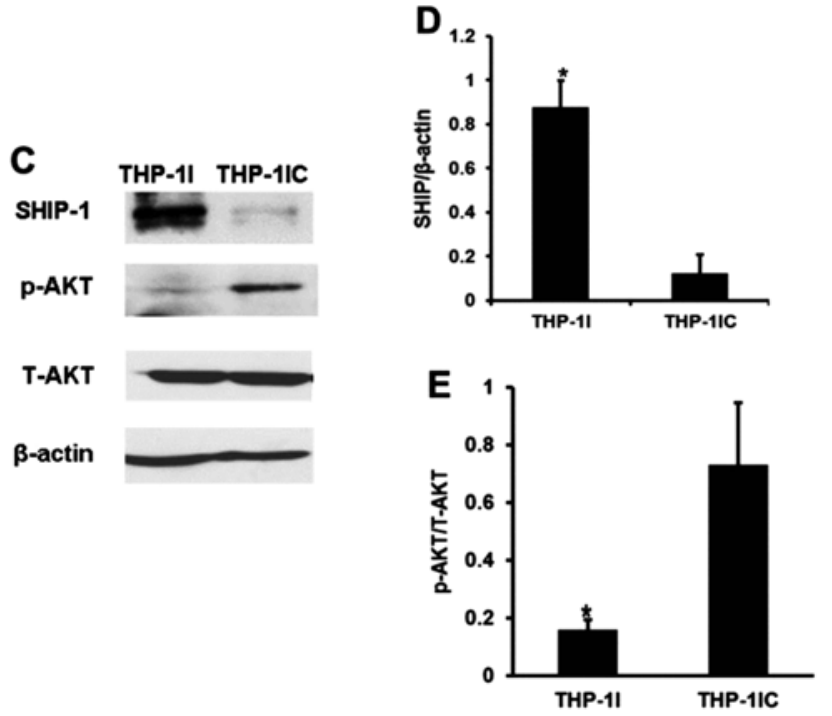

Figure 4. Expression of SHIP1, AKT, and p-AKT after transfection with the miR-155 inhibitor. Relative gene expression of (A) miR-155 and (B) SHIP1 at the mRNA level in THP-1 cells without transfection (THP-1c), and in THP-1 cells transfected with the miR-155 inhibitor (THP-1I) or with the miR-155 inhibitor control (THP-1Ic). (C) Protein levels of SHIP1, T-AKT and p-AKT in the THP-1I and THP-1Ic cells. (D) A significantly increased SHIP1 level and (E) decreased p-AKT/T-AKT ratio were found in the THP-1I cells when compared with the THP-1Ic cells. *P<0.05 vs. THP-1Ic.

the result of overexpression of BCR/ABL. Thus, the U937 and THP-1 cell lines were chosen for further study. Specifically, THP-1 cells expressed a higher miR-155 level but a lower SHIP1 level between the two cell lines studied (Fig. 2A). In contrast, U937 cells had a higher SHIP1 level but a lower miR-155 expression (Fig. 2B).

Expression of SHIPI, AKT and pAKT after miR-155 transfection. Since SHIP1 is a negative regulator of the PI3K/AKT signaling pathway, we hypothesized that increased activation of PI3K/AKT signaling in AML samples with low SHIP1 expression may be observed after miR-155 transfection. Because the levels of miR-155 expression were lower in the
U937 cells than that in the THP-1 cells, we chose to transfect miR-155 mimics into the U937 cells (Fig. 3A). Transfection of miRNA-155 mimics in the U937 cells did not alter the expression of SHIP1 at the mRNA level compared with the expression in the blank control and negative transfection groups ( $P>0.05$, Fig. 3B). However, U937 cells transfected with the miRNA155 mimics had significantly reduced expression of SHIP1 at the protein level $(\mathrm{P}<0.05$, Fig. $3 \mathrm{C}$ and $\mathrm{D})$, associated with unchanged AKT content but increased p-AKT protein levels $(\mathrm{P}<0.05$, Fig. $3 \mathrm{C}$ and E). Consequently, inhibition of SHIP1 expression by miR-155 would likely increase the activity of the AKT pathway. Therefore, miR-155 appears to behave as an onco-miR by reducing SHIP1 expression. 

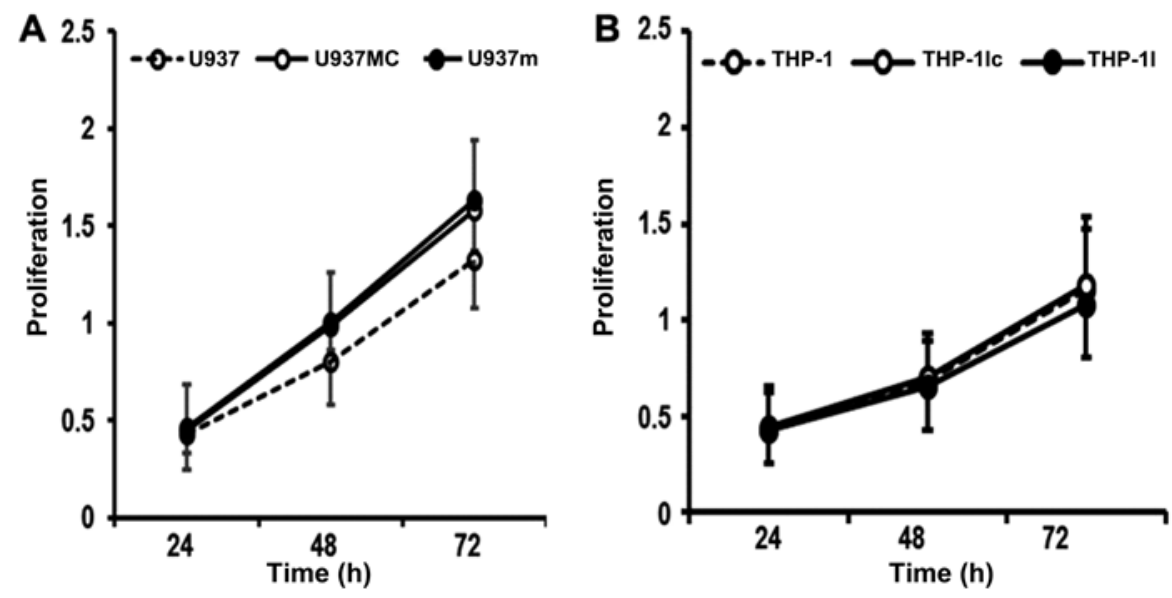

Figure 5. Proliferation in the cell lines after transfection. (A) Proliferation of the U937 cells without transfection (U937), or transfected with the miR-155 mimics (U937m), or with the mimic control (U937mc). (B) Proliferation of THP-1 cells without transfection (THP-1), or THP-1 cells transfected with the miR-155 siRNA (THP-1I), or with the miR-155 siRNA control (THP-1Ic).
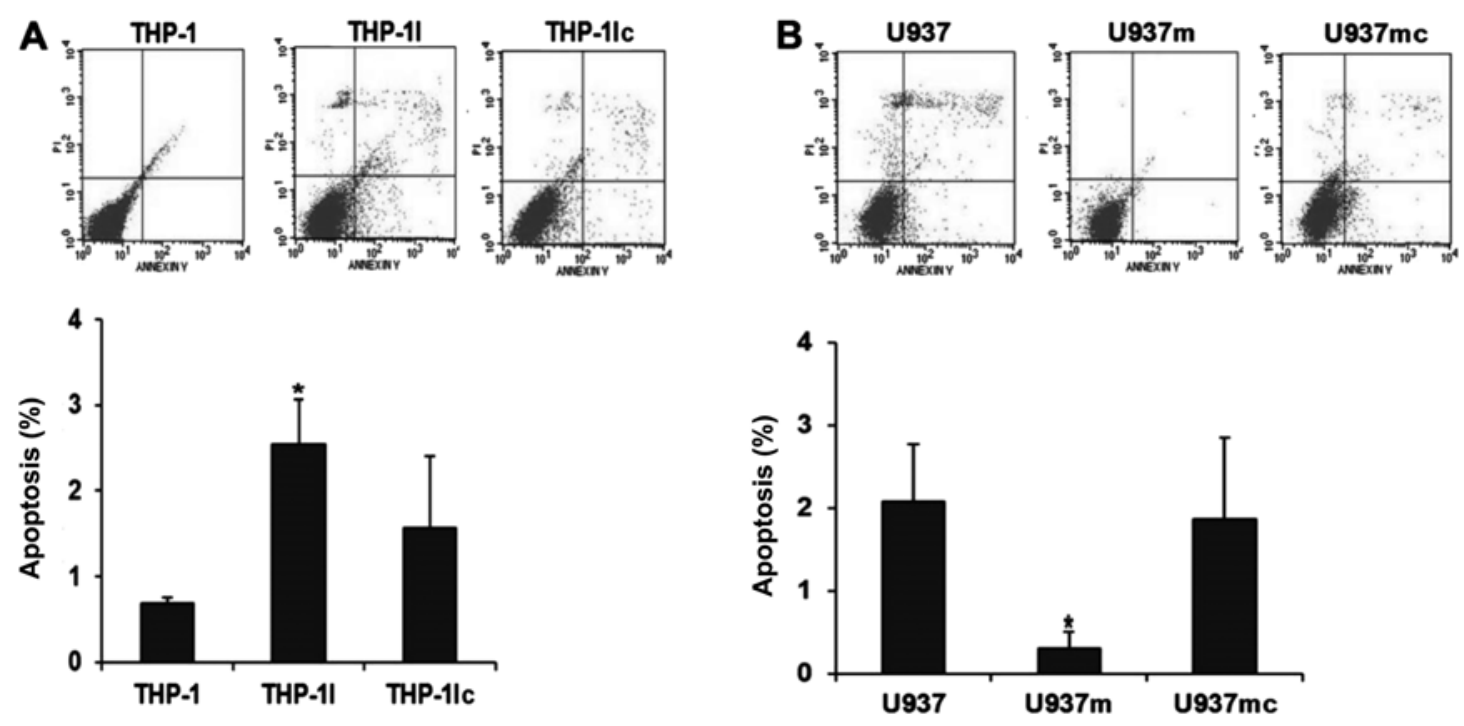

Figure 6. Apoptosis in the cell lines after transfection as measured by flow cytometry. (A) A significantly increased apoptosis rate was found in the THP-1I cells when compared to this rate in the THP-1c cells ( $\mathrm{P}<0.05)$, and (B) a significantly decreased apoptosis rate was found in the U937m cells when compared to this rate in the U937mc cells $\left({ }^{*} \mathrm{P}<0.05\right)$.

Expression of SHIP1, AKT, and pAKT after transfection of the miR-155 inhibitor. We then used miR-155 siRNA to examine the effects of restoring SHIP1 protein levels by reducing miR-155 expression in the THP-1 cells (Fig. 4A), which are known to overexpress miR-155. When THP-1 cells were transfected with the miR-155 siRNA, significantly elevated levels of SHIP1 protein were found, although no alterations in SHIP1 gene expression at the mRNA level could be detected compared with the expression levels in the blank and negative controls (Fig. 4B). THP-1 cells transfected with miR-155 siRNA also exhibited no alteration in total AKT content, but increased SHIP1 protein and decreased p-AKT levels were found $(\mathrm{P}<0.05$ and $\mathrm{P}<0.05$ respectively; Fig. $4 \mathrm{C}-\mathrm{E})$. It appears that suppression of miR-155 expression leads to inactivation of pAKT without altering total AKT via restoration of SHIP1 protein. Therefore, SHIP1 negatively regulates signaling in the PI3K/AKT pathway. SHIP1 could be a tumor suppressor.
Proliferation and apoptosis in the cell lines after transfection. To investigate the role of the miR-155/SHIP1/PI3K/AKT pathway in leukemogenesis, we performed proliferation and apoptosis assays in cell lines. First, we analyzed the effect on the autonomous proliferation of cells after transfection. Compared with the cells transfected with the mimic control (U937mc), the proliferation rate of the cells transfected with the miR-155 mimics (U937m) appeared higher but did not reach a statistically significant difference at any time point (U937m vs. U937mc; all P>0.05; Fig. 5A). However, the U937m cells had a significantly lower apoptosis rate when compared with that of the U937mc cells (U937m vs. U937mc; $\mathrm{P}<0.05$; Fig. 6A), indicating that miR-155 might cause AML progression by reducing cell apoptosis rather than increasing cell proliferation.

Similar results were also noted in the proliferation rate of THP-1 cells transfected with the miR-155 siRNA (THP-1I) 
and transfected with the inhibitor control (THP-1Ic), which indicated that miR-155 inhibition could not affect the proliferation rate of THP-1 at any time point (THP-1I vs. THP-1Ic; $\mathrm{P}>0.05$; Fig. 5B). A significantly increased rate of apoptosis was observed in the THP-1I cells compared with that of the THP-1Ic cells (THP-1I vs. THP-1Ic; P<0.05; Fig. 6B), indicating miR-155 inhibition may cause an increased apoptosis rate in AML without interfering with proliferation.

\section{Discussion}

Considering that SHIP1 is a negative regulator of the PI3K/AKT signaling pathway, inactivation of SHIP1 may result in Akt activation $(1,2,15)$. SHIP1-deficient mice develop myeloproliferative disease and leukemia $(11,16)$. Inactivating point mutations of SHIP1 have been observed in AML. It appears that SHIP1 mutations are an uncommon cause of the reduction in SHIP1 activity (7). In our study, low SHIP1 protein expression levels that did not completely correspond to SHIP1 mRNA levels were detected in a subset of patients with AML. We then tried to identify the molecular basis for the loss of SHIP1 function at the protein level.

microRNAs are a newly discovered class of short, noncoding RNA species, which may serve as master switches in gene networks $(8,17-20)$. miR-155 was shown to bind to the 3'UTR of SHIP1 mRNA and, therefore, to inhibit its translation $(9-11,16,20,21)$. SHIP1 is expressed in the same cell types that express miR-155, and it plays an opposing role in many cases. Some studies demonstrated a strong correlation between myeloproliferative disorders (MPDs) caused by miR-155 expression and specific knockdown of SHIP1 $(11,16)$. Recent studies showed that SHIP1 is a primary target of miR-155. Therefore, miR-155-mediated suppression of SHIP1 expression may contribute to the development of human MPDs and myeloid leukemia, in which miR-155 has been shown to be overexpressed $(8,16,19,20,22,23)$. Moreover, it was recently reported that the endogenous expression of SHIP1 is reduced in hematopoietic cells overexpressing miR-155 in vitro and in vivo, which leads to increased activation of AKT (3). Our present findings are consistent with these reports.

We overexpressed miR-155 in U937 cells and found that the levels of SHIP1 protein were largely reduced in these cells. Furthermore, in THP-1 cells, we inhibited miR-155 expression using siRNA targeting miR-155 and found that the level of SHIP1 protein was increased. Following recent studies showing that SHIP1 plays an important role in the pathogenesis of lymphoma and acute lymphoblastic leukemia that is affected by miR-155 $(3,14)$, we investigated the existence of an association between SHIP1 and miR-155 in AML. Our results demonstrated that SHIP1 is also a direct target of miR-155 in AML cells. We hypothesized that inhibition of the translation of SHIP1 mRNA by miR-155 could be an important mechanism for the loss of function of SHIP1.

To evaluate the importance of SHIP1/miR-155 in AML, we initially examined the levels of expression of SHIP1 and miR-155 in myeloid leukemia cells. We hypothesized that reduced expression of SHIP1 in leukemia cells could be a result of an elevated miR-155 expression as in other hematological malignancies $(2,3,13,14,24)$. In fact, the levels of SHIP1 protein were lower in THP-1 and U937 cells than in control cells. In contrast, miR-155 levels were relatively higher. These results indicate that the expression of miR-155 is negatively associated with the expression of SHIP1 in AML cell lines. To test whether this observation could be found in patients with AML, we examined the expression of SHIP1 and miR-155 in $30 \mathrm{AML}$ patients. We found elevated levels of miR-155 in patients with acute myelomonocytic leukemia and acute monocytic leukemia, corresponding to the FAB-AML-M4 and FAB-AML-M5 classifications, respectively, as well as decreased expression of SHIP1 protein. These results indicate that miR-155 expression is inversely correlated with SHIP1 expression in myeloid leukemia cell lines and in M4 and M5 patients, suggesting that an interaction of SHIP1 and miR-155 may be biologically significant in these two subtypes of AML. In addition, SHIP1 expression is downregulated in AML, whereas miR-155 is commonly overexpressed in subsets of AML patients and acts as an onco-miR. It is likely that the downregulation of SHIP1 expression by miR-155 is a plausible mechanism of miR-155-mediated oncogenesis in AML M4 or M5. This study found an association between the level of miR-155 and a specific leukemic phenotype, similar to the finding that miR-181 expression is positively correlated with M1 and M2 subtypes of AML (25), suggesting a possible role in the developmental lineage and differentiation state of the tumors $(12,20-23,26,27)$.

Given that miR-155 is overexpressed in AML and that it may act as an onco-miR, we decided to examine whether miR-155 has oncogenic functions in AML cells in vitro. Our results indicated that miR-155 may cause AML by reducing cell apoptosis rather than increasing cell proliferation. The results of our in vitro assays indicated that miR-155 mimics inhibited the apoptosis of U937 cells. Conversely, transfection of anti-miR-155 in THP-1 cells increased apoptosis. Western blot analyses confirmed that the expression of SHIP1 in these cells was modulated by miR-155. Taken together, our results showed that miR-155 acts as an onco-miR in AML cells by downregulating SHIP1 expression. In addition, SHIP1 was also shown to be a tumor suppressor that regulates cell apoptosis in AML.

SHIP1 has been known to be a negative feedback regulator of PI3K/Akt signaling, and the antitumoral activity of SHIP1 is mediated by inhibition of Akt signaling via the PI3K pathway $(1,2,4,5,15,28-30)$. Akt activation may affect many cellular processes, including cell cycle progression, transcription, translation, differentiation, and apoptosis. Given that SHIP1 expression is inhibited by miR-155, we hypothesized that miR-155 overexpression in AML may play a role in Akt signaling. In U937 cells with overexpressed miR-155, Akt was found to be activated. Conversely, we found that antimiR-155 reduced p-Akt in THP- 1 cells. Meanwhile, the levels of Akt protein were not affected by miR-155 overexpression or anti-miR-155. These results demonstrated that overexpression of miR-155 enhanced oncogenic Akt signaling via the SHIP1/PI3K pathway in AML cells. Given that the expression of miR-155 was significantly enhanced and that Akt signaling was active in the AML cell lines and a subset of patients with AML, we propose that the miR-155/SHIP1/PI3K/Akt pathway may play an important role in the pathogenesis of AML.

In summary, we hypothesized that inhibition of the expression of SHIP1 could be a mechanism by which miR-155 acts 
as an onco-miR in leukemia. Overexpression of miR-155 alters AKT signaling by inhibiting SHIP1 expression. The severe adverse effects and limitations of conventional chemotherapy and hematopoietic stem cell transplantation underscore the urgent need for new treatment strategies for patients with AML. Our findings provide new insight into the pathogenesis of leukemia and suggest that restoration of SHIP1 expression as a tumor suppressor by inhibiting miR-155 may represent a useful approach for the treatment of certain subtypes of AML.

\section{Acknowledgements}

This research was supported by the Special Research Fund of the Ministry of Health of China (no. 201202017) and the Natural Science Foundation of Hebei Province (no. H2012206139).

\section{References}

1. Hamilton MJ, Ho VW, Kuroda E, et al: Role of SHIP in cancer. Exp Hematol 39: 2-13, 2011.

2. Conde C, Gloire G and Piette J: Enzymatic and non-enzymatic activities of SHIP-1 in signal transduction and cancer. Biochem Pharmacol 82: 1320-1334, 2011.

3. Costinean S, Sandhu SK, Pedersen IM, et al: Src homology 2 domain-containing inositol-5-phosphatase and CCAAT enhancer-binding protein beta are targeted by miR-155 in B cells of Emicro-MiR-155 transgenic mice. Blood 114: 1374-1382, 2009.

4. Kennah M, Yau TY, Nodwell M, et al: Activation of SHIP via a small molecule agonist kills multiple myeloma cells. Exp Hematol 37: 1274-1283, 2009.

5. Lo TC, Barnhill LM, Kim Y, Nakae EA, Yu AL and Diccianni MB: Inactivation of SHIP1 in T-cell acute lymphoblastic leukemia due to mutation and extensive alternative splicing. Leuk Res 33: 1562-1566, 2009.

6. Luo JM, Yoshida H, Komura S, et al: Possible dominant-negative mutation of the SHIP gene in acute myeloid leukemia. Leukemia 17: 1-8, 2003.

7. Gilby DC, Goodeve AC, Winship PR, Valk PJ, Delwel R and Reilly JT: Gene structure, expression profiling and mutation analysis of the tumour suppressor SHIP1 in Caucasian acute myeloid leukaemia. Leukemia 21: 2390-2393, 2007.

8. O'Connell RM, Zhao JL and Rao DS: MicroRNA function in myeloid biology. Blood 118: 2960-2969, 2011.

9. Teng G and Papavasiliou FN: Shhh! Silencing by microRNA-155. Philos Trans R Soc Lond B Biol Sci 364: 631-637, 2009.

10. Tili E, Croce CM and Michaille JJ: miR-155: on the crosstalk between inflammation and cancer. Int Rev Immunol 28: 264-284, 2009.

11. O'Connell RM, Chaudhuri AA, Rao DS and Baltimore D: Inositol phosphatase SHIP1 is a primary target of miR-155. Proc Natl Acad Sci USA 106: 7113-7118, 2009.

12. Schwind S, Maharry K, Radmacher MD, et al: Prognostic significance of expression of a single microRNA, miR-181a, in cytogenetically normal acute myeloid leukemia: a Cancer and Leukemia Group B study. J Clin Oncol 28: 5257-5264, 2010.
13. Pedersen IM, Otero D, Kao E, et al: Onco-miR-155 targets SHIP1 to promote TNFalpha-dependent growth of B cell lymphomas. EMBO Mol Med 1: 288-295, 2009.

14. Yamanaka Y, Tagawa H, Takahashi N, et al: Aberrant overexpression of microRNAs activate AKT signaling via down-regulation of tumor suppressors in natural killer-cell lymphoma/leukemia. Blood 114: 3265-3275, 2009.

15. Metzner A, Precht C, Fehse B, et al: Reduced proliferation of CD34(+) cells from patients with acute myeloid leukemia after gene transfer of INPP5D. Gene Ther 16: 570-573, 2009.

16. O'Connell RM, Rao DS, Chaudhuri AA, et al: Sustained expression of microRNA-155 in hematopoietic stem cells causes a myeloproliferative disorder. J Exp Med 205: 585-594, 2008.

17. Sayed D and Abdellatif M: AKT-ing via microRNA. Cell Cycle 9: 3213-3217, 2010.

18. Volinia S, Galasso M, Costinean S, et al: Reprogramming of miRNA networks in cancer and leukemia. Genome Res 20: 589-599, 2010

19. Zhi F, Cao X, Xie X, et al: Identification of circulating microRNAs as potential biomarkers for detecting acute myeloid leukemia. PLoS One 8: e56718, 2013.

20. Vasilatou D, Papageorgiou S, Pappa V, Papageorgiou E and Dervenoulas J: The role of microRNAs in normal and malignant hematopoiesis. Eur J Haematol 84: 1-16, 2010.

21. Faraoni I, Antonetti FR, Cardone J and Bonmassar E: miR-155 gene: a typical multifunctional microRNA. Biochim Biophys Acta 1792: 497-505, 2009.

22. Wang Y, Li Z, He C, et al: MicroRNAs expression signatures are associated with lineage and survival in acute leukemias. Blood Cells Mol Dis 44: 191-197, 2010.

23. Yuan Y, Kasar S, Underbayev C, Prakash S and Raveche E: MicroRNAs in acute myeloid leukemia and other blood disorders. Leuk Res Treatment 2012: 603830, 2012.

24. Lawrie CH: MicroRNAs and haematology: small molecules, big function. Br J Haematol 137: 503-512, 2007.

25. Debernardi S, Skoulakis S, Molloy G, Chaplin T, Dixon-McIver A and Young BD: MicroRNA miR-181a correlates with morphological sub-class of acute myeloid leukaemia and the expression of its target genes in global genome-wide analysis. Leukemia 21: 912-916, 2007

26. Hyde RK and Liu PP: The role of microRNAs in acute myeloid leukemia. F1000 Biol Rep 2: 81, 2010.

27. Joyce CE and Novina CD: miR-155 in acute myeloid leukemia: not merely a prognostic marker? J Clin Oncol 31: 2219-2221, 2013

28. Baran CP, Tridandapani S, Helgason CD, Humphries RK, Krystal G and Marsh CB: The inositol 5'-phosphatase SHIP-1 and the Src kinase Lyn negatively regulate macrophage colony-stimulating factor-induced Akt activity. J Biol Chem 278: 38628-38636, 2003.

29. Blunt MD and Ward SG: Targeting PI3K isoforms and SHIP in the immune system: new therapeutics for inflammation and leukemia. Curr Opin Pharmacol 12: 444-451, 2012.

30. Ong CJ, Ming-Lum A, Nodwell M, et al: Small-molecule agonists of SHIP1 inhibit the phosphoinositide 3-kinase pathway in hematopoietic cells. Blood 110: 1942-1949, 2007. 